\title{
Front-End electronics and integration of ATLAS pixel modules
}

\author{
F. Hügging ${ }^{1}$ \\ on behalf of the ATLAS Pixel Collaboration[1] \\ Universität Bonn, Physikalisches Institut \\ Nußallee 12, D-53115 Bonn, Germany
}

\begin{abstract}
For the ATLAS Pixel Detector fast readout electronics has been successfully developed and tested. Main attention was given to the ability to detect small charges in the order of 5,000 $e^{-}$within 25 ns in the harsh radiation environment of LHC together with the challenge to cope with the huge amount of data generated by the 80 millions channels of the Pixel detector. For the integration of the $50 \mu \mathrm{m}$ pitch hybrid pixel detector reliable bump bonding techniques using either lead-tin or indium bumps has been developed and has been successfully tested for large scale production.
\end{abstract}

Key words: atlas, silicon detector, pixel, front-end electronics, deep sub-micron, hybridization, bump-bonding

PACS: 06.60.Mr, 29.40.Gx

\section{Introduction}

The Pixel Detector of the ATLAS experiment at the LHC, which is currently under construction at CERN, Geneva, is the crucial part for good secondary vertex resolution and high b-tagging capability. Therefore high spatial resolution and fast read-out in a multi-hit environment is needed and leads to the use of a hybrid silicon pixel detector. This detector will consist of three barrel layers and three disks in each forward direction to have at least three space points up to $|\eta|=2.5$ for particles with a transversal momentum greater than $500 \mathrm{MeV}[1]$.

$\overline{1}$ phone: $+49(228) 733210$, e-mail: huegging@physik.uni-bonn.de 
The smallest detector unit will be a module made of one silicon pixel sensor and sixteen readout chips connected with high density bump bonding techniques to the sensor. Each module consists of about 50,000 pixel cells with the size of $50 \mu \mathrm{m} \cdot 400 \mu \mathrm{m}$ to reach the required spatial resolution of $12 \mu \mathrm{m}$ in $r \phi$-direction. The main requirement for the ATLAS pixel modules is high radiation tolerance of all components in a harsch radiation environment close to the interaction point. The integrated design fluence is $10^{15} \mathrm{~cm}^{-2} 1 \mathrm{MeV}$ neutron equivalent coming predominantly from charged hadrons over ten years for the outer barrel layers and disks and five years for the innermost layer. Secondly the total need of about 1,800 modules requires very good testability of all components before assembly and high fault tolerance in long term operation as they are not supposed to be exchanged during the whole foreseen ten years lifetime of the ATLAS experiment. The material budget for the whole pixel detector is very strict to affect the later detector parts as less as possible. This leads to a total amount of material less than $1.2 \%$ of one radiation length per module not including further services and support structures.

\section{Module concept}

A cross-section of an ATLAS pixel module can be seen in figure 1. The module basically consists of a so called bare module which meant the sixteen readout chips bump bonded to the silicon pixel sensor. The size of the module is roughly given by the size of the sensor $18.6 \cdot 63.0 \mathrm{~mm}^{2}$. The readout chips with are placed in two rows of eight. The front-end chips are slightly longer than the sensor width to be able to reach the wire-bond pads of the chips. The interconnection techniques using fine pitch bump bonding is either done with $\mathrm{Pb} / \mathrm{Sn}$ by $\mathrm{IZM}^{2}$ or with Indium by $\mathrm{AMS}^{3}$. The total active area of one module is $16.4 \cdot 60.8 \mathrm{~mm}^{2}$ with 46080 pixel.

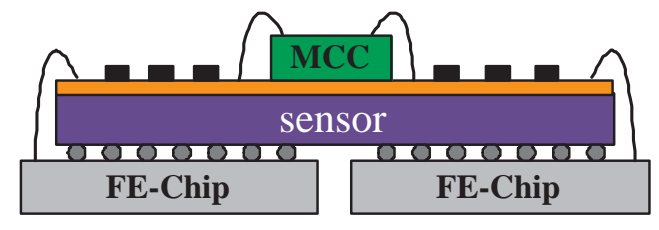

Fig. 1. Cross-section of an ATLAS pixel module.

For routing the signals to the MCC [2] and further to off-module electronics and providing the analog and digital supply powers to the readout chips a two layer high density interconnect (flexible capton cable) is used. Also the flexible hybrid carries the MCC chip which performs control and clock operations. It is glued to backside of the sensor and has roughly the size of the sensor tile. To

$\overline{2}$ Institut für Zuverlässigkeit und Mikrointegration, Berlin, Germany.

3 Alenia Marconi Systems, Roma, Italy. 
connect the flex hybrid with the Front-End chips and with the MCC standard wire bond techniques are used; altogether around 350 wire-bonds are needed for a complete module. The module is glued chips down to the mechanical support which also serves as cooling structure. The whole power consumption of about $4 \mathrm{~W}$ per module has to be dissipated through the readout chips to allow an operation of the module at $-6^{\circ} \mathrm{C}$.

\section{Front-End electronics}

Each FE-chip contains of 2880 pixel cells arranged in 18 columns times 160 rows. Every pixel cell consists a charge sensitive amplifier, a discriminator for zero suppression and a digital readout logic to transport the hit information to the periphery. The signal charge can be determined by measuring the width of the discriminator output signal. Hits are temporarily stored in one of the 64 buffers per column pair located at the chip periphery until a trigger signal selects them for readout. Hits with no corresponding trigger signal are discarded after the programmable trigger latency of up to 256 clock cycles of $25 \mathrm{~ns}$. All hits corresponding to trigger are sent through one serial LVDS link to module controller chip which builds full modules events containing hit information of all 16 FE chips on a module.

Figure 2 shows a block diagram of most important elements in the pixel cell. Charge deposited in the sensor is brought to the pixel through the bump bond pad which connects to the input of a charge sensitive amplifier. An inverting folded cascode amplifier is fed back by a capacitor of $C_{f}=6.5 \mathrm{fF}$ integrated into the lower metal layers of the bump bond pad. The feedback capacitor is discharged by a constant current so that a triangular pulse shape is obtained. As consequence of this particular pulse shape, the width of the discriminator output signal is nearly proportional to the deposited charge. This signal is called 'time over threshold' or ToT, is measured in units of the bunch crossing clocks (25 ns) and provides an analogue information of evry hit with a resolution of 4-6 bit. The feedback current $I_{f}$ is set globally with an 8 bit on-chip bias DAC so that a compromise between a good ToT resolution and small dead time in the pixel can be found. The constant feedback current generation is part of a circuit which compensates for detector leakage current. This must be sourced by the electronics because the pixel sensor is dc-coupled to readout chip. The compensation circuit can cope with more than $100 \mathrm{nA}$ detector leakage current per pixel.

The threshold generation of each discriminator is done within every single pixel. A small threshold dispersion between the 2880 pixel is crucial in order to achieve low threshold settings without an increased noise hit rate. Therefore a number of 7 individually programmable trim bits in each pixel covers a wide 


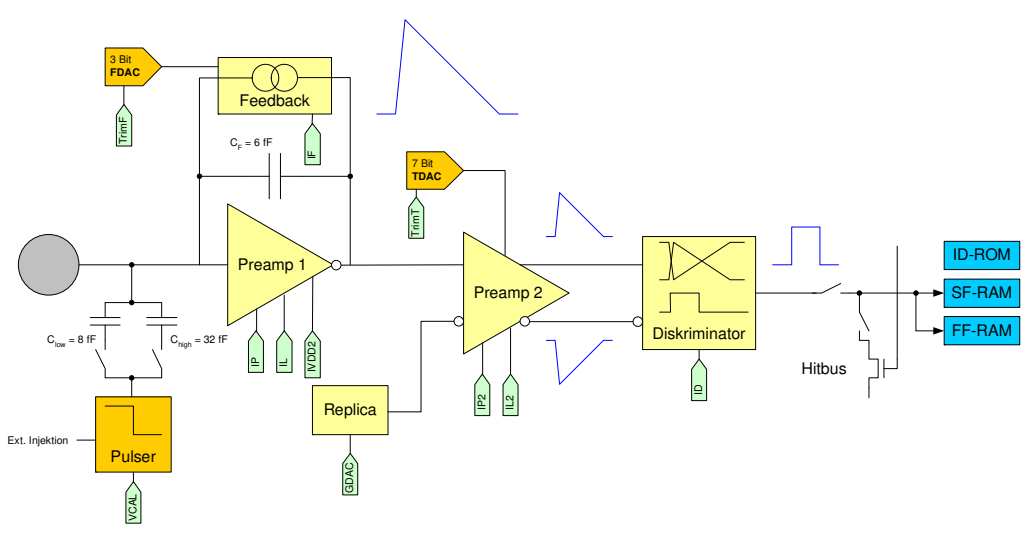

Fig. 2. Block diagram of the pixel unit cell. The output signal of a charge sensitive amplifier is compared to a threshold voltage to detect hits. The time of the rising and falling edge of the discriminator is recorded.

range of thresholds between $1,000 e^{-}$and $10,000 e^{-}$. These local trim bits affects both side of a differential pair amplifier leading to a linear threshold vs. DAC behaviour in the central part of the covered threshold range. Furthermore a globally set 5 bit DAC, so called GDAC, allows to move the threshold for the whole chip without losing the triming between the individual pixel.

Every pixel contains a calibration circuit used to inject known charges into the input node. This circuit generates a voltage step which is connected to one of two injection capacitors for different charge ranges.

The output signal of the discriminator in every pixel can be disabled with a local mask bit. A fast OR of a programmable subset of pixel is available for testing purposes and for self-triggered operation of the chip by using this OR signal after delaying it as a trigger. The 14 configuration bits used in every pixel are stored in SEU tolerant DICE cells [4] which can be written and read back by means of a shift register running vertically through the column. The layout of this cell has been significantly changed with respect to the previous chip (FE-I1) in order to improve the SEU tolerance. For the globally set DACs a basic triple redundancy scheme with majority readback is used.

The basic philosophy of the readout is to associate the hit to unique bunch crossing by recording a time stamp when the rising edge of the discriminator occurs. The time of the falling edge is memorized as well in order to calculate the ToT as the difference of the two values in units of the bunch crossing clock. The two 8 bit RAM cells used for this purpose are classical static memory cells.

The readout scheme can be divided into four elementary tasks running on the chip in parallel. First time stamps generated by an 8 bit Gray counter and clocked with bunch crossing clock are stored for the rising and falling edge of the discriminators in the pixel. After receiving the falling edge a hit flag is set and the pixel is ready to be processed. Secondly as soon as hits are 
flagged to the Column Control Logic the uppermost hit pixel is requested to send its rising and falling edge time stamps together with its ID down the column where is information is stored in a free location of the End-of-Column $(\mathrm{EoC})$ buffer pool. The pixel is then cleared and the scan continues the search for other hit pixel. Thirdly the hit stays in the EoC buffer until the trigger latency has elapsed. The leading edge time stamp of the hits in the buffer are therefore permanently compared to the actual time stamp minus the fix but programmable latency. When the comparison is true and a trigger signal is present the hit is flagged as valid for readout, it is discarded otherwise. Incoming triggers are counted on the chip, the trigger number is stored together with the flag in the EoC buffer so that several trigger do not lead to confusion. A list of pending triggers is kept in a FIFO. Lastly a Readout Controller initiates the serial readout of hit data as soon as pending triggers are present in the Trigger FIFO, the EoC buffers are searched for valid data with correct trigger numbers. The column and row address and the ToT of these hits are serialized and send to the MCC. The Readout Controller adds a start-of-event and an end-of-event word together with error and status bits to the data stream.

The recent chip (FE-I2) has been designed in a quarter micron technology with 6 metal layers using radiation tolerant layout rules [3]. Tests of the first batches received back from the vendor from May 2003 on showed that the design is fully functional except one problem dealing with race condition in the control block leading to the need to operate the chip with a reduced digital supply voltage of $1.6 \mathrm{~V}$ instead of the nominal $2.0 \mathrm{~V}$. But this problem has been fixed via a re-distribution of the clock signal by changing 2 metal layers slightly in the back-end processing (FE-I2.1).

\section{Results of prototype modules}

Several ten modules have been built with older chip generation (FE-I1) in order to qualify the bumping process and the whole module production chain. As an example of the overall good quality of these modules one can see in figure 3 the hitmap and spectrum obtained with one module illuminated by an $\mathrm{Am}^{241}$-source from the top using the self-trigger possibility of the chip. Only less than 10 out of 46,080 pixel don't see source hits showing the excellent bump quality of this module which is true for almost all modules. For every channel the ToT response was calibrated individually using the integrated charge generation circuit. Afterwards this calibration was applied to the source measurement in order to obtain the shown spectrum which is a sum over all pixel. Since no clustering of hit data was applied the spectrum is relatively broad but one can clearly see the main $60 \mathrm{keV}$ photo-peak roughly at the expected value of 16,600 $e^{-}$. More results obtained with FE-I1 modules can be found in [5]. 


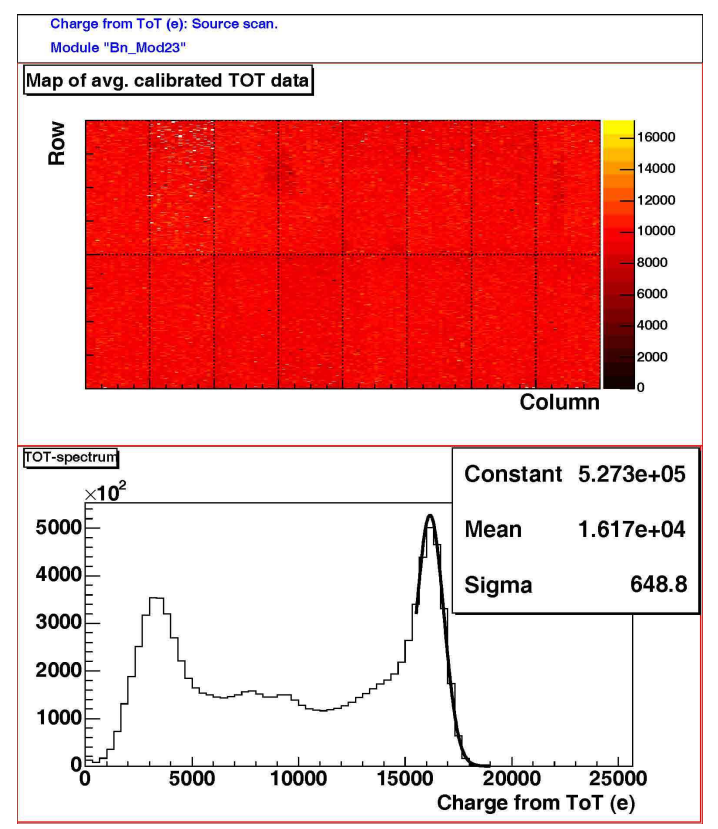

Fig. 3. Am ${ }^{241}$-Spectrum measured with an ATLAS pixel module using the self-trigger capabilities and the ToT charge information. Each channel of the module has been individually calibrated and the shown spectrum is a sum over all pixel without any clustering.

Recently first modules with the newer chip generation (FE-I2.1) has been built. Figure 4 and 5 show the threshold distribution and noise measured for a whole module after the trimming of the individual pixel to a threshold of roughly $3,100 e^{-}$. All these measurements were done with internal charge injection circuit. One can see that the reached overall threshold dispersion is only $40 e^{-}$which has to be compared to value of $100 e^{-}$reached by the older chip generation. Note further that there is no single pixel with an threshold lower than 2,900 $e^{-}$. This shows the high tuning capability of this chip which is important to reach small thresholds on the whole module without any extra noisy pixel especially after irradiation.

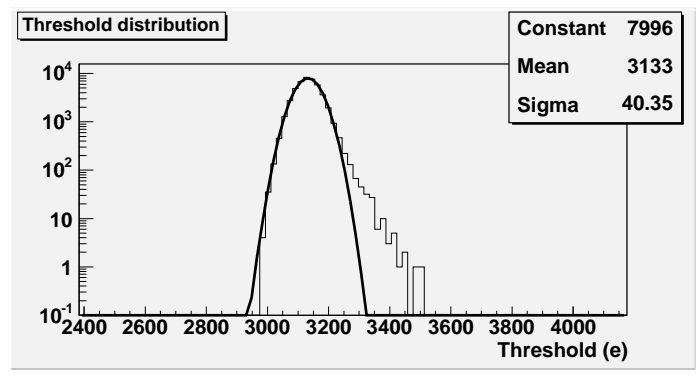

Fig. 4. Threshold distribution of a module built with FE-I2.1 chips after the tuning procedure.

The measured mean noise of the module is with $150 e^{-}$for the standard pixel much better than the specification of $300 e^{-}$, even the longer and so called ganged pixel located in the inter-chip regions which show due to the higher 
input capacity a higher noise of $170 e^{-}$and $270 e^{-}$resp. are well below this specification.

The most demanding requirement of LHC is the time correct hit detection within one bunch crossing cycle ( $25 \mathrm{~ns}$ ) together with the strict power budget of less than $4 \mathrm{~W}$ for the whole 16 chip module. Therefore not the real threshold is important but the threshold taken only hits into account which arrive within in time slot of $20 \mathrm{~ns}$ after the injection. Using a special delay circuit inside the MCC measurements with the needed accuracy are possible and in figure 6 the results are shown for the same module tuned to a threshold of $3,100 \mathrm{e}^{-}$. Because the timing is very sensitive to the input capacity of the amplifier the in-time-threshold is slightly different for the different kind of pixel. But overall a in-time-threshold of 4,200 $e^{-}$is reached and also the special pixel don't show a threshold higher than $4,600 e^{-}$meaning that only an extra charge of $1,100 e^{-}$and 1,500 $e^{-}$resp. is needed to meet the timing requirement of ATLAS. This results are very much improved with respect to the previous chip generation where the in-time-threshold was in the order of 5,000-6,000 $e^{-}$by a tuned threshold of $3,000 e^{-}$. This exceeds specification of $5,000 e^{-}$given by the fact that after irradiation damage only half of the initial charge of $20,000 e^{-}$is available.

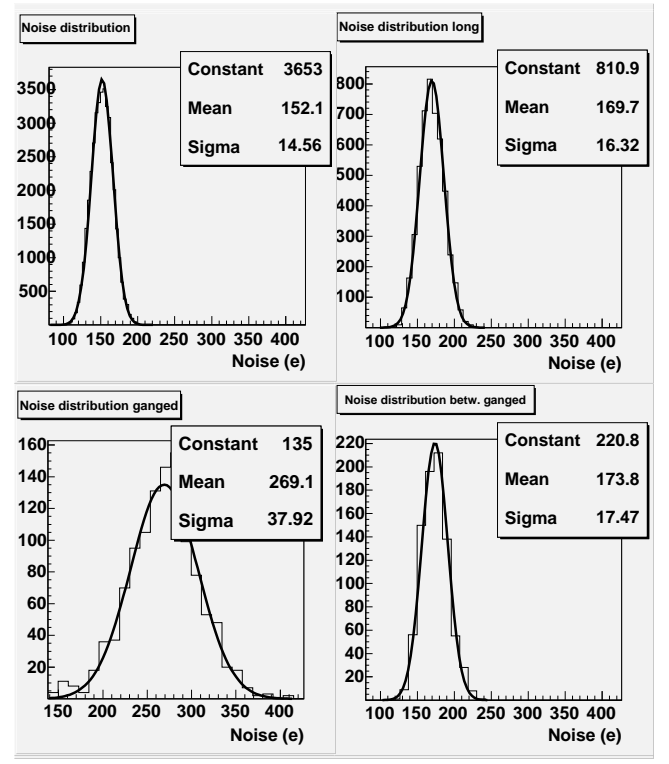

Fig. 5. Noise distributions for the different pixel types of a module built with FE-I2.1 chips after the tuning procedure.

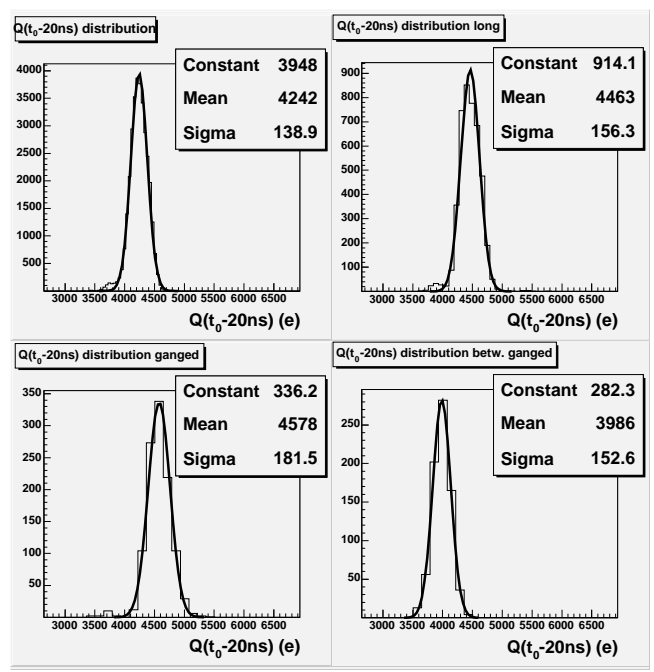

Fig. 6. In-time-threshold distributions for the different pixel types of a module built with FE-I2.1 chips tuned to an average threshold of $3,100 e^{-}$

More extensive studies have been done with these modules, including beam test measurements and measurements during and after proton irradiation up to $100 \mathrm{MRad}$ or $2 \cdot 10^{15} \mathrm{n}_{e q} \mathrm{~cm}^{-2}$ well above the design fluence of $50 \mathrm{MRad}$ and $1 \cdot 10^{15} \mathrm{n}_{e q} \mathrm{~cm}^{-2}$ respectively. All these measurement confirmed the good 
quality of the chip and the whole module, in particular it was shown that these modules meet all requirements and are able to operate in the environment of ATLAS and LHC.

\section{Conclusions}

The recent ATLAS pixel Frond-End chip generation has been successfully produced, assembled to full size modules and tested. Improvements concerning the timing behaviour and chip threshold tuning capability with respect to the previous chip generation has been obtained. All further results including beam tests and irradiation confirmed the overall good quality of the chip reaching all requirements. By building more than 100 modules with high quality a lot of experience has been gained with the fine pitch bump bonding process for pixel module using two different vendors and technologies showing that the target of building 2,000 modules needed for ATLAS in the next two years is feasible.

\section{References}

[1] ATLAS Pixel Detector Technical Design Report, CERN LHCC 98-13 (1998).

[2] R. Beccherle et al., Nucl. Instr. and Meth. A492 (2002) 117-133.

[3] W. Snoeys et al., Nucl. Instr. and Meth. A439 (2000) 349-360.

[4] T. Calin, M. Nicolaidis, R. Velazco, IEEE Trans. Nucl. Science, Vol. 43, No. 6, Dec. 1996, 2874-2878.

[5] J. Grosse-Knetter, this proceedings. 